\section{Mit Brachytherapie später mehr Lebensqualität}

Bei Prostatakarzinom mit günstigem Risiko gelten radikale Prostatektomie und Brachytherapie als vergleichbar effektiv. Umso wichtiger, die Auswirkungen auf die spätere Lebensqualität in die Entscheidung einzubeziehen.

Z ehn Jahre nach radikaler Prostatektomie oder Brachytherapie sind jeweils mehr als $90 \%$ der Patienten am Leben. Bei Prostatakarzinom mit günstigem Risiko (Gleason-Score $\leq 6$, PSA $<10$, Stadium T1-T2a) bietet man den Patienten im Allgemeinen beides an. Allerdings sind belastbare klinische Daten, mit welcher Methode die Patienten in puncto Lebensqualität langfristig besser fahren, bisher Mangelware. Die US-Studie SPIRIT (Surgical Prostatectomy Versus Interstitial Radiation Intervention Trial) sollte dem abhelfen, musste aber wegen Rekrutierungsproblemen nach zwei Jahren abgebrochen werden. Dennoch liefert sie wichtige Hinweise zur Lebensqualität. Insgesamt 168 Patienten machten median

\section{Harn-Inkontinenz ist unter Brachytherapie weniger häufig als unter radikaler Prostatektomie}

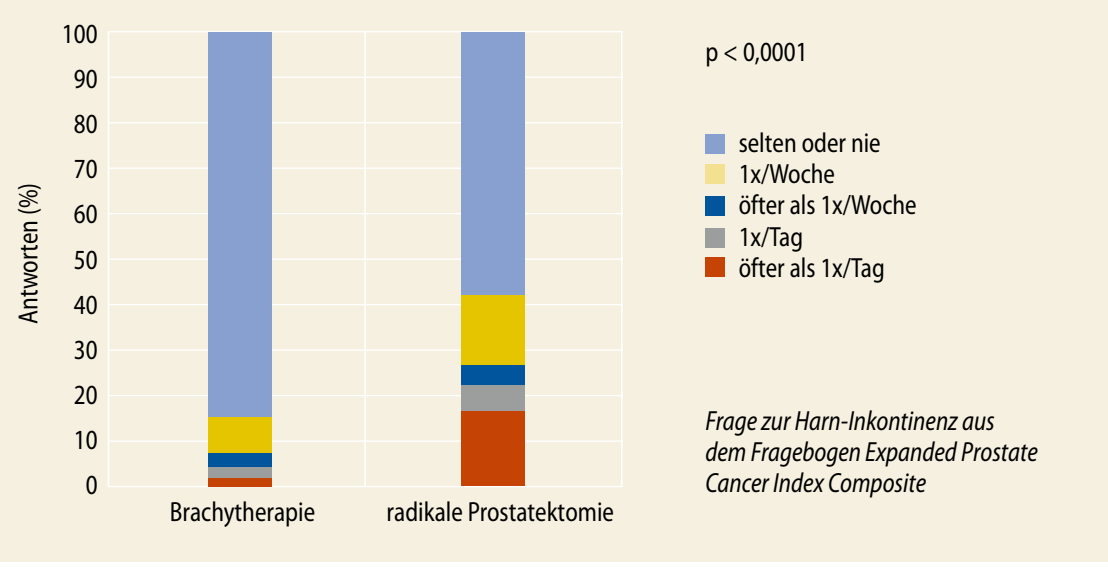

\section{Neuer Androgensynthese-Hemmer bei kastrationsresistentem Prostatakarzinom}

\begin{abstract}
Abirateron ist ein neuer oraler Inhibitor der Androgensynthese. Im April 2011 wurde die Substanz von der US-Arzneibehörde FDA für die Therapie des kastrationsresistenten Prostatakarzinoms zugelassen. Ein internationales Onkologenteam hat nun in einem Review-Artikel die Daten der wichtigsten Studien gesichtet und versucht, den Stellenwert von Abirateron genauer zu bestimmen.
\end{abstract}

W ächst ein metastasiertes Prostatakarzinom auch unter Androgendeprivation weiter, wird in erster und zweiter Linie auf eine Docetaxel- oder Cabazitaxel-basierte Therapie zurückgegriffen. Angesichts der zwar signifikanten, dennoch mäßigen Erfolge geht indes die Suche nach einfach $\mathrm{zu}$ verabreichenden Substanzen weiter. Idealerweise sollten sie
5,3 Jahre nach Therapie Angaben zur gesundheitsbezogenen Lebensqualität. Alle hatten bei Rekrutierung an einer Informationssitzung teilgenommen und sich danach für eine Randomisierung (32 Patienten) oder direkt für Resektion ( $\mathrm{n}=50)$ bzw. Strahlentherapie $(n=86)$ entschieden. Von den befragten Patienten hatten $60,7 \%$ eine Brachytherapie hinter sich, $39,3 \%$ eine radikale Prostatektomie. Die bestrahlten Patienten waren median etwas älter als die operierten.

Hinsichtlich der gesundheitsbezogenen Lebensqualität, erhoben 3,2 bis 6,5 Jahre nach der Intervention, zeigte die Brachytherapie signifikante Vorteile bei den Domänen, die Harnentleerung und Erektionsfähigkeit betrafen. Außerdem war die Zufriedenheit der Patienten nach Brachytherapie signifikant größer. Dagegen fanden sich keine Unterschiede zwischen beiden Gruppen, wenn es um den Darm und die hormonelle Situation ging.

Fazit: Für die spätere Lebensqualität scheint die Brachytherapie günstiger zu sein - vor allem hinsichtlich Harnsymptomen, Erektionsfähigkeit und Patientenzufriedenheit.

Ulrike Wepner

Crook JM et al. Comparison of health-related quality of life 5 years after SPIRIT: surgical Prostatectomy versus Interstitial Radiation Intervention Trial. J Clin Oncol. 2011;29:362-8 an Position 17, das zum Beispiel für die Synthese von Dehydroepiandrosteron und Androstendion wichtig ist.

Einen Meilenstein setzte die Substanz in einer Phase-III-Studie, deren Ergebnisse erstmals im Herbst vergangenen Jahres auf dem Treffen der European Society of Medical Oncology vorgestellt wurden. Abirateron in einer Dosierung von $1.000 \mathrm{mg} / \mathrm{d}$ war darin mit Placebo - jeweils plus 2x 5 mg Prednison/d - verglichen worden. Die knapp 1.200 Probanden waren zuvor mit Docetaxel behandelt worden, die Progression konnte dadurch jedoch nicht aufgehalten werden. Die mediane Überlebenszeit verlängerte sich unter Abirateron um $25 \%$ von zwölf auf 15 Monate. Das zuständige Komitee stoppte daraufhin die Studie, die Patienten des Placeboarms erhielten ebenfalls Abirateron. Die Verträglichkeit 